\title{
Multiple extended target tracking algorithm based on GM-PHD filter and spectral clustering
}

\author{
Jinlong Yang ${ }^{*}$, Fengmei Liu, Hongwei Ge and Yunhao Yuan
}

\begin{abstract}
With the increase of the resolution of modern radars and other detection equipments, one target may produce more than one measurement. Such targets are referred to as extended targets. Recently, multiple extended target tracking (METT) has drawn a considerable attention. However, one crucial problem is how to partition the measurement sets accurately and rapidly. In this paper, an improved METT algorithm is proposed based on the Gaussian mixture probability hypothesis density (GM-PHD) filter and an effective partition method using spectral clustering technique. First, the density analysis technique is introduced to eliminate the disturbance of clutter, and then the spectral clustering technique based on neighbor propagation is used to partition the measurements. Finally, the GM-PHD filter is implemented to achieve the METT. Simulation results show that the proposed algorithm has a better performance, especially a better real-time performance, than the conventional distance partition and K-means++ methods.
\end{abstract}

Keywords: Extended target; Measurement partition; Probability hypothesis density; Spectral clustering

\section{Introduction}

In most target tracking cases, it is assumed that one target generates at most one measurement per time step due to the low resolution sensor system, i.e., each target is tracked as a single point source, and its extension is assumed to be neglectable in comparison with sensor resolution [1-3]. However, with the increase of the resolution of modern radars and other detection equipments, the echo signal of a target may be distributed in a different range resolution cell, so the measurement is no longer equivalent to a point, i.e., a single target may produce multiple measurements. Such target is referred to as an extended target in [4-7]. Recently, extended target tracking is a hot topic in the field of data fusion and has drawn a considerable attention, especially the multiple extendedtarget tracking (METT) [8-14].

In the conventional extended target tracking, the measurements are modeled as a spatial distribution model in [4], and two examples are used to prove the effectiveness of the approach: a point target with more measurement sources and an object with infinitely thin stick of length $l$. Poisson process with a spatially dependent rate

\footnotetext{
* Correspondence: yjlgedeng@163.com

School of Internet of Things Engineering, Jiangnan University, Wuxi 214122, China
}

parameter is introduced in [5], assuming that each target produces measurements with Poisson-distributed random number. It is considered that in this measurement model, the target is sufficiently far away from the sensor, and the measurements resemble a point cluster rather than a geometric structure [9-11]. Random matrix $(\mathrm{RM})$ is proposed in [15], which has been used to track elliptical target extension [12-14,16,17]. Different methods to estimate star-convex target extension and other general shapes are the random hypersurface model (RHM) and star-convex RHM by Baum and Hanebeck $[6,7]$. However, these methods can only effectively achieve the single extended target tracking, and they cannot track the multiple extended targets with unknown and varying number.

Recently, the random finite set (RFS) method $[18,19]$ for multi-target tracking has proven to be useful, which allows estimation of multiple targets in the presence of false measurements and detection uncertainty in a Bayesian filter framework [20-22]. Especially, Mahler has extended the probability hypothesis density (PHD) filter to the METT [8] and derived a complete updated formula, which can effectively achieve the METT. Gaussian mixture (GM) technique is introduced to yield a proximate solution in $[9,10]$, and the proposed algorithm 
is referred to as extended target (ET)-GM-PHD filter. Cardinality PHD (CPHD) filter and random matrix technique are proposed to improve the performance of METT in $[11,13]$. However, the problem of how to effectively partition the measurements of the extended multi-targets remains unresolved in these methods. The partition method based on distance is proposed in [10], which needs suitable thresholds to generate enough partitions including the correct partition. So, the number of partitions grows rapidly as the target number increases, which implies that the distance partition consumes a large computational time, making the tracking algorithm intractable. The K-means ++ method, the predicted partition method, and the ML method are proposed in [12], but they also cannot effectively achieve the measurement partition.

As to the difficulty of METT in measurement partition, a novel measurement partition algorithm is proposed based on spectral clustering technique under the ET-GM-PHD framework. First, the clutter can be effectively eliminated from the measurement set by the density analysis. Then, the spectral clustering technique is used to achieve the measurement partition quickly. Finally, the ET-GM-PHD filter is implemented to obtain the states of the extended targets. Simulation results show that the proposed method has a better performance in measurement set partition than the conventional methods.

The rest of the paper is organized as follows. Section 2 summarizes the measurement partition and the multiple extended target PHD filter. Section 3 presents the proposed measurement partition algorithm based on the Gaussian kernel density analysis and the spectral clustering and then implements the ET-GM-PHD filter. In Section 4, two examples are presented with simulation results to compare the performance of the proposed method with those of the conventional methods. Finally, the conclusions are given in Section 5.

\section{Backgrounds}

\subsection{Measurement partition}

In the METT, measurement partition is a matter of prime importance due to many measurements originating from a single target. The aim is to keep the measurements originating from a single target in the same class. However, the number of target is varying with time, and there is much clutter in the tracking scenario, which makes the measurements hard to be partitioned accurately.

Assume at time $k$, there are three measurements in the measurement set $Z_{k}=\left\{z_{k}^{(1)}, z_{k}^{(2)}, z_{k}^{(3)}\right\}$, then the partition problem can be described as follows [10],

$$
\begin{aligned}
& P_{1}: W_{1}^{1}=\left\{z_{k}^{(1)}, z_{k}^{(2)}, z_{k}^{(3)}\right\} \\
& P_{2}: W_{1}^{2}=\left\{z_{k}^{(1)}, z_{k}^{(2)}\right\}, W_{2}^{2}=\left\{z_{k}^{(3)}\right\} \\
& P_{3}: W_{1}^{3}=\left\{z_{k}^{(1)}, z_{k}^{(3)}\right\}, W_{2}^{3}=\left\{z_{k}^{(2)}\right\} \\
& P_{4}: W_{1}^{4}=\left\{z_{k}^{(2)}, z_{k}^{(3)}\right\}, W_{2}^{4}=\left\{z_{k}^{(1)}\right\} \\
& P_{5}: W_{1}^{5}=\left\{z_{k}^{(1)}\right\}, W_{2}^{5}=\left\{z_{k}^{(2)}\right\}, W_{3}^{5}=\left\{z_{k}^{(3)}\right\}
\end{aligned}
$$

where $P_{i}$ denotes the $i$ th partition, and $W_{j}^{i}$ denotes the $j$ th subset in the $i$ th partition, $\left|P_{i}\right|$ denotes the subset number in the $i$ th partition, $\left|W_{j}^{i}\right|$ denotes the measurement number of the $j$ th subset in the $i$ th partition.

\subsubsection{Distance partition theorem}

Let $d($.$) be a distance measure between each pair of mea-$ surements, and $d_{l} \geq 0$ be an arbitrary distance threshold. Then, there is only one partition in which any pair of measurements $z_{k}^{(i)}$ and $z_{k}^{(j)}$ satisfying $d\left(z_{k}^{(i)}, z_{k}^{(j)}\right) \leq d_{l}$ are in the same cell $[10,12]$.

It is noted that different distance thresholds may produce different partitions; therefore, many different thresholds are selected for producing enough partitions including the correct one in the distance partition algorithm, which makes this method time-consuming.

\subsection{Multiple extended target PHD filter}

The standard PHD filter for single measurement target tracking has been described in [19]; however, it is not suitable for METT. Recently, Mahler has derived a correct equation for extended target PHD filter based on the Poisson multi-target measurement model [8]. The predicted formula is the same as the standard PHD filter [19]. The updated formula for the METT can be described as [8]

$$
D_{k \mid k}(x)=L_{Z_{k}}(x) D_{k \mid k-1}(x)
$$

where $D_{k \mid k}(x)$ denotes an intensity function of the state $x$, and $L_{Z_{k}}(x)$ denotes the pseudo likelihood function. $Z_{k}$ denotes the measurement set at time $k$. When $Z_{k}=\varnothing$,

$$
L_{Z_{k}}(x) \triangleq 1-p_{D}(x)+e^{-r(x)} p_{D}(x)
$$

otherwise,

$$
\begin{aligned}
& L_{Z_{k}}(x) \triangleq 1-\left(1-e^{-r(x)}\right) p_{D}(x) \\
& \quad+e^{-r(x)} p_{D}(x) \sum_{P \angle Z_{k}} \omega_{P} \sum_{W \in P} \frac{r(x)^{|W|}}{d_{W}} \prod_{z \in Z} \frac{\phi_{z}(x)}{\lambda_{k} c_{k}}
\end{aligned}
$$

where $P \angle Z_{k}$ denotes a partition subset $P$ of the measurement set $Z_{k}, W$ denotes a subset of a partition $P$, and $\cup_{W \in P} W=Z_{k} \cdot \phi_{z}(x)$ denotes the measurement likelihood function of one measurement originating 
from an extended target $x, r(x)$ is the measurement expectation, and $p_{D}(x)$ denotes the detection probability of the sensor. Clutter has a Poisson distribution, and its density can be described as $\kappa_{k}\left(z_{k}\right)=\lambda_{k} c_{k}\left(z_{k}\right), \lambda_{k}$ denotes the mean number of clutter measurements, and $c_{k}\left(z_{k}\right)$ is the space distribution of the clutter.

$$
\begin{aligned}
& \omega_{P}=\frac{\prod_{W \in P} d_{W}}{\sum_{P^{\prime} \angle Z_{k}} \prod_{W \in P^{\prime}} d_{W^{\prime}}} \\
& d_{W}=\delta_{|W|, 1}+D_{k \mid k-1}\left[e^{-r} r^{|W|} p_{d} \prod_{z \in W} \frac{\phi_{z}}{\lambda_{k} c_{k}}\right]
\end{aligned}
$$

where $\delta_{i, j}$ denotes the Kronecker delta function and $r=r(x)$.

The Gaussian mixture implement of the multiple extended target PHD filter is presented in [10] and referred to as ET-GM-PHD filter.

\section{The proposed algorithm}

In order to solve the problem of the measurement partition, a novel method is proposed in this section. The spectral clustering technique is introduced to partition the measurements of the multiple extended targets; then, the ET-GM-PHD framework is employed to estimate the target states. However, the spectral clustering method is sensitive to the clutter. To solve this problem, the clutter measurements are eliminated from the measurement sets by the Gaussian kernel density analysis technique.

\subsection{Gaussian kernel density}

Assume $x_{1}, x_{2}, \ldots, x_{n} \subset R^{d}$ are independent and identically distributed (i.i.d) random variables, whose probability distribution function is defined as $f(x)$, then the kernel density estimator $\hat{f}(x)$ can be obtained by [23,24]:

$$
\hat{f}(x)=\frac{1}{n h^{d}} \sum_{i=1}^{n} K\left(\frac{x_{i}-x}{h}\right)
$$

where $K(\cdot)$ denotes the kernel function, and $h$ denotes the bandwidth (BW) parameter of the kernel. Moreover, the kernel function must satisfy the following properties: $K(-u)=K(u), \operatorname{Sup}|K(u)|<\infty$, and $\int_{-\infty}^{+\infty} K(u) d u=1$. Generally, kernel functions include Gaussian function, Epanechnikov function, and biweight function. In this paper, assume that $Z_{k}=\left\{z_{k}^{i}\right\}_{i=1}^{N_{z, k}}$ denotes the measurement set at time $k$ and the Gaussian function is used as the kernel function. Then, the Gaussian kernel density function of the measurements can be described as

$$
f\left(z_{k}^{i}\right)=\frac{1}{N_{z, k} h^{d / 2}(2 \pi)^{d / 2}} \sum_{j=1}^{N_{z, k}} \exp \left(-\frac{1}{2 h}\left(z_{k}^{i}-z_{k}^{j}\right)\left(z_{k}^{i}-z_{k}^{j}\right)^{T}\right)
$$

where $N_{z, k}$ denotes the number of measurements at time $k$, and $T$ denotes the transpose operator. $z_{k}^{i}$ denotes the $i$ th measurement of the measurement set $Z_{k}$.

In order to decrease the computational cost, only the neighbor measurements are considered for density computation. Therefore, we define the measurement density as

$$
f\left(z_{k}^{j}\right)=\frac{1}{C} \sum_{z_{k}^{i} \in \text { near }\left(z_{k}^{j}\right)} \exp \left(-\frac{d\left(z_{k}^{j}, z_{k}^{i}\right)^{2}}{2 h}\right)
$$

where $z_{k}^{i} \in n e a r\left(z_{k}^{j}\right)$ means that the $z_{k}^{i}$ and $z_{k}^{j}$ are neighbors,and $d\left(z_{k}^{j}, z_{k}^{i}\right)$ denotes the Euclidean distance between $z_{k}^{j}$ and $z_{k}^{i}$. $C$ is the normalization constant. As can be seen from Equation 9, the more focused the measurements are, the bigger the measurement densities will be.

Figure 1 shows the measurement density of the extended target and clutter measurements. As can be seen that if a suitable density threshold is selected, the clutter measurements can be removed effectively.

\subsection{Density analysis and clutter elimination}

In this section, the density threshold $\tau$ is selected by density histogram, and used to remove the clutter from the measurement sets. The density threshold can be obtained as follows.

(1) Assume that $f_{\max }$ and $f_{\text {min }}$ denote the maximum measurement density and the minimum measurement density, respectively. Divide $\left[f_{\min } f_{\text {max }}\right]$ into $N_{z}$ equal parts, where $N_{z}$ denotes the number of the measurements. The density interval can be described as $\left[f_{\min } f_{\min }+d\right),\left[f_{\min }+d f_{\min }+2 d\right), \ldots,\left[f_{\max }-d f_{\max }\right]$, where $d=\left(f_{\max }-f_{\min }\right) / N_{z}$.

(2) Calculate the measurement number of each density interval, find out the first density interval which does not include any measurement ranging from the maximum density to the minimum density, and then each density value in this interval can be selected as the density threshold $\tau$. In this paper, the maximum density value in this interval is selected.

Notice that there are more measurements in the area of high density. On the contrary, small measurements exist in area of low density, which may be clutters. Therefore, a density interval without measurements may exist, which is the interval to be obtained. 


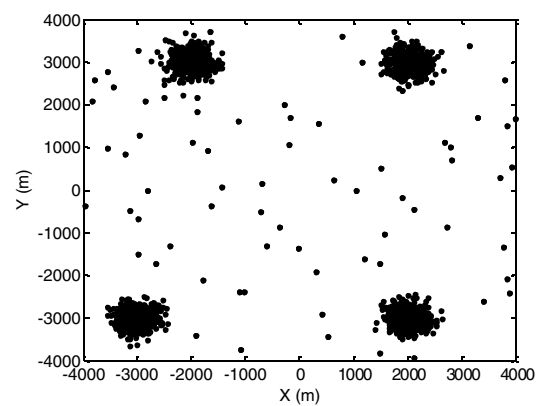

(a)

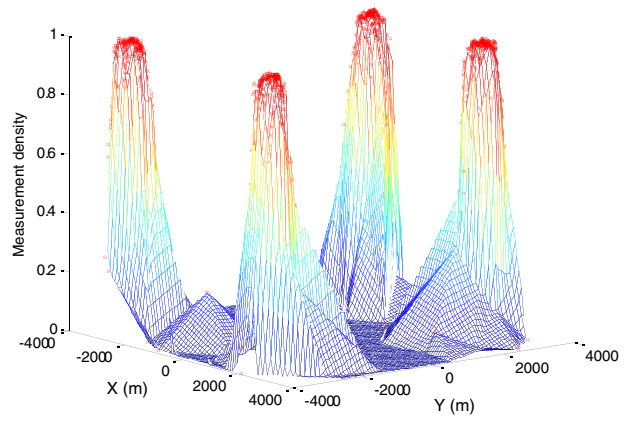

(b)

Figure 1 Measurement density. (a) Measurements of the targets and clutter. (b) Measurement density of the targets and clutter.

\subsubsection{Clutter removal}

If $f\left(z_{k}^{i}\right)>\tau$, then set $z_{k}^{i}$ as the measurement originating from the target; otherwise, set $z_{k}^{i}$ as the clutter measurement and remove it from the measurement set. Finally, the new measurement set without clutter can be obtained and described as $G_{k}=\left\{z_{k}^{i} \mid f\left(z_{k}^{i}\right)>\tau\right\}$.

\subsection{Spectral clustering based on neighbor propagation}

In recent years, spectral clustering has become one of the popular clustering approaches due to their high performance in data clustering and simplicity in implementation [25]. Compared with traditional clustering techniques, there is no need to suppose the data distribution as spheral in spectral clustering method; thus, the nonspheral distributed clusters can be recognized by using the eigenvectors of the normalized similarity matrix. Assume at time $k$, the measurement set without clutter is $G_{k}=\left\{z_{k}^{i}\right\}_{i=1}^{n}$, the spectral clustering process based on neighbor propagation is described as follows $[25,26]$.

(1) Calculate the similarity matrix $C$ of the new measurement set $G_{k}$.

(a) Calculate each Euclidean distance between two measurements, i.e. $b_{i j}=\sqrt{\left(z_{k, x}^{i}-z_{k, x}^{j}\right)^{2}+\left(z_{k, y}^{i}-z_{k, y}^{j}\right)^{2}}$, and construct the distance matrix $B=\left[b_{i j}\right]_{n \times n}$, where $\left(z_{k, x}^{i}, z_{k, y}^{i}\right)$ denotes the position of the $i$ th measurement, and $n=\left|G_{k}\right|$ denotes the measurement number in $G_{k}$. Then the similarity matrix can be denoted as $C=\left[c_{i j}\right]_{n \times}$ ${ }_{n}$, where $c_{i j}=\exp \left(-\frac{b_{i j}^{2}}{2 \sigma^{2}}\right), \sigma$ is a scale parameter to control how fast the similarity attenuates with the distance between the $i$ th and $j$ th measurements [26]. In this paper, we set $\sigma$ as an empirical parameter, and let $\sigma=1.5$. (b) The neighbor relation matrix is defined as $T=\left[t_{i j}\right]$ $n \times n$, which is a symmetrical matrix. In the initial stage, set each element value as zero, and if $b_{i j}$ $<\varepsilon$, let $t_{i j}=1$ and $t_{j i}=1$, where $\varepsilon=\max _{i=1: n}$ $\left(\min _{j=1: n}\left(b_{i j}\right)\right)$ denotes the distance threshold.

(c) Calculate the mode matrix $M$ according to the rule of neighbor propagation and the neighbor relation matrix $T$. The rule of neighbor propagation can be described as, if $\left(g_{k}^{i}, g_{k}^{j}\right) \in R$ and $\left(g_{k}^{j}, g_{k}^{l}\right) \in R$, then $\left(g_{k}^{i}, g_{k}^{l}\right) \in R$.

The mode $M_{i}$ denotes the $i$ th row of $M$, and the measurement subset corresponding to the mode $M_{i}$ meets the neighbor relation. $|M|$ denotes the number of the modes.

(d) Update $T$ and $C$. If $t_{i j}=1, t_{j i}=1$, and $t_{i k}=0$, set $t_{i k}=1, t_{k i}=1, c_{i k}=\min \left(c_{i j}, c_{j k}\right)$, and $c_{k i}=c_{i k}$. Update the similarity of each mode, let $c(m, n)$ $=\max \left(\min \left(C\left(M_{i}, M_{j}\right)\right)\right)$, where $m$ and $n$ denote the measurements of $M_{i}$ and $M_{j}$, respectively.

(2) Calculate the Laplacian matrix $L, L=D^{-1 / 2} C D^{-1 / 2}$, where $D$ is a diagonal matrix, and $D_{i i}=\sum_{j=1}^{n} C_{i j}$, $D_{i j}=0, i \neq j$.

(3) Construct the matrix $V$. Let $V=\left[v_{1}, v_{2}, \ldots, v_{K}\right]$, where $v_{1}, v_{2}, \ldots, v_{K}$ are the eigenvectors corresponding to the $K$ largest eigenvalues $1=\lambda_{1} \geq \lambda_{2}, \ldots, \geq \lambda_{K}$ of the matrix $L$.

(4) Form the matrix $Y$ from $V$ by renormalizing each row of $V$, and the element $y_{i j}$ of $Y$ is defined as $y_{i j}=V_{i j} /\left(\sum_{j=1}^{K} V_{i j}^{2}\right)^{1 / 2}$.

(5) Set each row of $Y$ as a sample of the $K$ - dimension space, and use the K-means++ method to cluster the samples. Then the samples are divided into $K$ classes, where $K$ is an integer and $K \in\left(K_{L}, K_{U}\right) . K_{L}$ and $K_{U}$ denote the minimum and the maximum 
class numbers, respectively. Set $\operatorname{Class}(K)$ as a cluster result of $G_{k}$.

(6) Repeat the step (5) to obtain the measurement partition $P_{k}=\underset{K \in\left(K_{L}, K_{U}\right)}{\cup} \operatorname{Class}(K)$.

\subsubsection{Remark 1}

Assume that $N_{G}$ is the number of measurements in $G_{k}$ and $\beta$ denotes the measurement rate conforming to the Poisson distribution. Then, the mean and the variance of the measurement rate are identical and equal to $\beta$. Therefore, $2 \beta$ measurements are produced at most from an extended target, so we can set $K_{L}=\left\lceil\frac{N_{G}}{2 \beta}\right\rceil$. Moreover, | $M \mid$ is bigger than the number of the targets, and is smaller than the measurement number, so we can set $K_{U}=|M|$.

\subsection{The ET-GM-PHD filter implementation}

Implement the ET-GM-PHD filter according to the measurement partition $P_{k}$, and extract the target states. Refer to [10] for the detailed process.

\section{Simulations}

In order to demonstrate the performance of the proposed algorithm, we implement the proposed measurement partition method, the distance partition, and the K-means++ method under the framework of ET-GM-PHD filter [10]. The two examples are implemented on Dell computer with Intel(R) Core(TM) CPU i5 3470, 3.2 GHz and 4GB RAM (Dell Inc., Round Rock, TX, USA).

Two different metrics are used for evaluating the algorithm performance. The first is the statistics of the target number estimates, and the second is the optimal subpattern assignment (OSPA) distance [27], which is recently developed and defined as

$$
\bar{d}_{p}^{(c)}(X, Y)=\left(\frac{1}{n}\left(\min _{\pi \in \prod_{n}} \sum_{i=1}^{m} d^{(c)}\left(\mathbf{x}_{i}, \mathbf{y}_{\pi(i)}\right)^{p}+c^{p}(n-m)\right)\right)^{1 / p}
$$

where $X=\left\{\mathbf{x}_{1}, \cdots, \mathbf{x}_{m}\right\}$ and $Y=\left\{\mathbf{y}_{1}, \cdots, \mathbf{y}_{n}\right\}$ are arbitrary finite subsets, $1 \leq p<\infty, c>0, m, n \in N_{o}=\{0,1,2, \cdots\}$. If $m$ $>n$, then $\bar{d}_{p}^{(c)}(X, Y)=\bar{d}_{p}^{(c)}(Y, X)$. In the simulation, the parameters of OSPA distance are set as $p=2$ and $c=60$. 100 Monte Carlo runs are performed.

\subsection{Example 1: crossing extended target tracking}

Assume that $X_{k}=\left[x_{k}, y_{k}, v_{x, k}, v_{y, k}\right]^{\mathrm{T}}$ denotes the extended target state at time $k$, where $\left(x_{k}, y_{k}\right)$ denotes the target position and $\left(v_{x, k}, v_{y, k}\right)$ denotes the velocity. $z_{k}^{(j)}=\left[x_{k}^{(j)}, y_{k}^{(j)}\right]$ is the measurement of the $j$ th sensor. Each extended target follows a linear Gaussian dynamical model, and sensor has a linear Gaussian measurement model, i.e.,

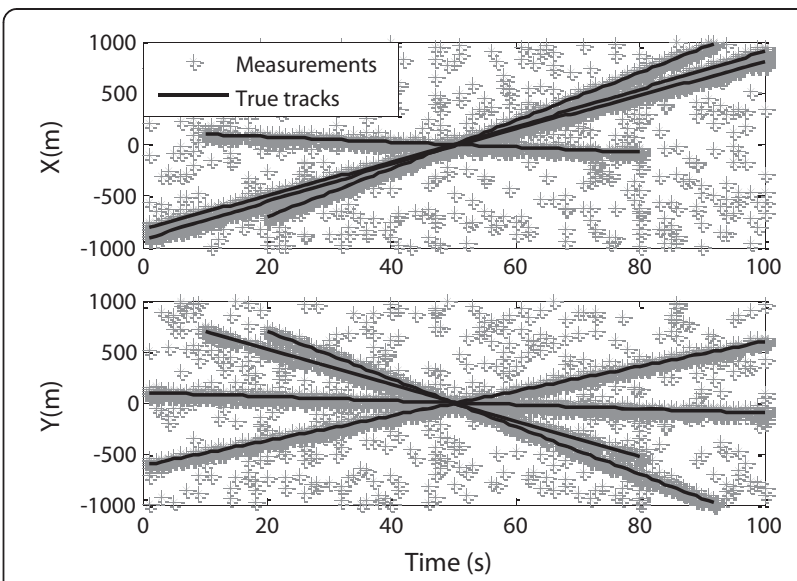

Figure 2 True tracks and the real measurements.

$$
\begin{aligned}
& f_{k \mid k-1\left(x_{k} \mid x_{k-1}\right)=N\left(x_{k} ; F x_{k-1}, Q_{k-1}\right)} \\
& g_{k}\left(z_{k} \mid x_{k}\right)=N\left(z_{k} ; H x_{k}, R_{k}\right)
\end{aligned}
$$

where $N(\cdot ; m, P)$ denotes a Gaussian density with mean $m$ and covariance $P . F=\left[\begin{array}{cc}I_{2} & \Delta t I_{2} \\ 0_{2} & I_{2}\end{array}\right]$ is the state transition matrix, and $Q_{k-1}=\sigma_{v}^{2}\left[\begin{array}{cc}\frac{\Delta t^{4}}{4} I_{2} & \frac{\Delta t^{3}}{2} I_{2} \\ \frac{\Delta t^{3}}{2} I_{2} & \Delta t^{2} I_{2}\end{array}\right]$ is the process noise covariance, where $I_{2}$ and $0_{2}$ denote the $2 \times 2$ identity and zero matrices, respectively. $\sigma_{v}=2$ is the standard deviation of the process noise, and $\Delta t=1 \mathrm{~s}$ is the sample interval. $H_{k}=\left[\begin{array}{ll}I_{2} & 0_{2}\end{array}\right]$ denotes the measurement matrix, $R_{k}=\sigma_{\varepsilon}^{2} I_{2}$ is the covariance of the measurement noise, and $\sigma_{\varepsilon}=20$ is the standard deviation of the measurement noise. Let the probabilities of target survival and detection be $P_{s}=0.99$ and $P_{D}=0.99$,

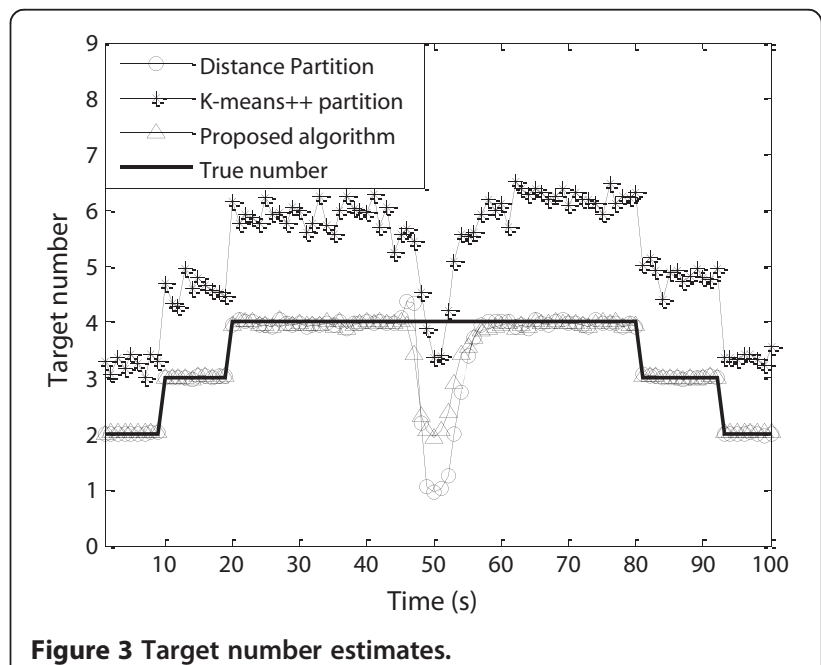




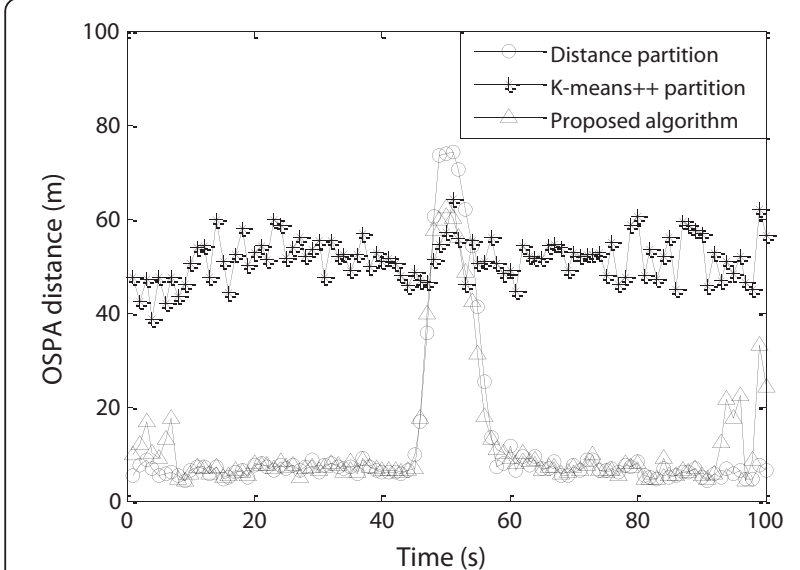

Figure 4 OSPA distance statistic.

respectively. The clutter is modeled as a Poisson RFS with the mean $\lambda=10$ over the observation space.

The birth intensity is described as

$$
\begin{aligned}
D_{b}(x)= & 0.1 N\left(x ; m_{b}^{(1)}, P_{b}\right)+0.1 N\left(x ; m_{b}^{(2)}, P_{b}\right) \\
& +0.1 N\left(x ; m_{b}^{(3)}, P_{b}\right)+0.1 N\left(x ; m_{b}^{(4)}, P_{b}\right)
\end{aligned}
$$

where $m_{b}^{(1)}=\left[\begin{array}{lllll}-800 & -800 & 0 & 0\end{array}\right]^{T}, m_{b}^{(2)}=\left[\begin{array}{lllll}-800 & -300 & 0 & 0\end{array}\right]^{T}$, $m_{b}^{(3)}=\left[\begin{array}{llll}-492 & 230 & 0 & 0\end{array}\right]^{T}, m_{b}^{(4)}=\left[\begin{array}{lllll}-654 & 409 & 0 & 0\end{array}\right]^{T}$, and $P_{b}=$ $\operatorname{diag}([100,100.25,25])$.

Figure 2 shows the real tracks of the extended targets with cluttered measurements in $\mathrm{x}$ - and $\mathrm{y}$-coordinates. Figure 3 shows the number estimate of the extended targets, and Figure 4 shows the OSPA distance. As can be seen, the proposed method has an accuracy similar to that of the distance partition method, but better than the $\mathrm{K}$-means++ method, which is mainly because the $\mathrm{K}$ means++ method is sensitive to the clutter and needs a good initial cluster center.

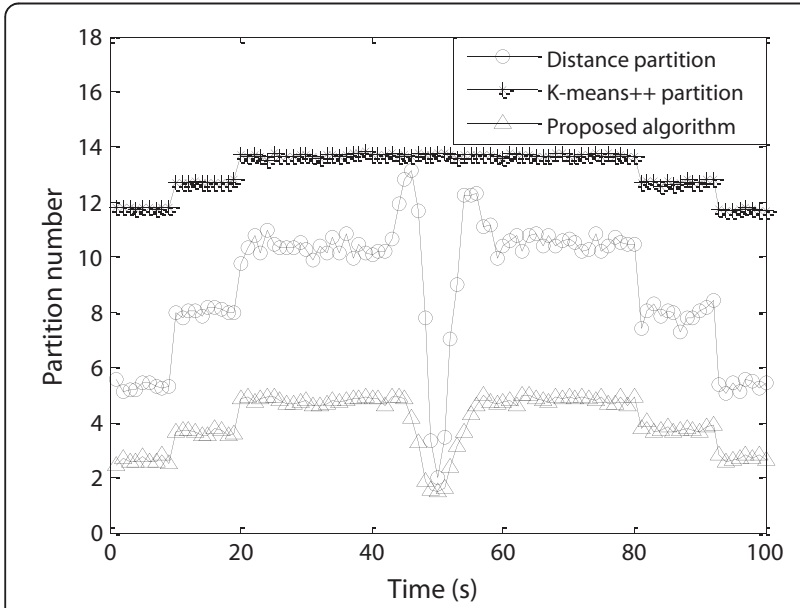

Figure 5 Partition number estimates.

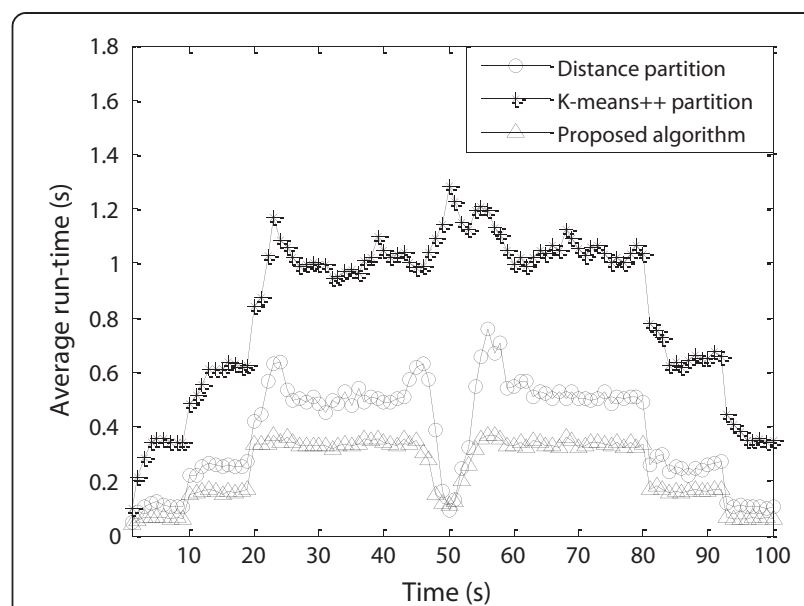

Figure 6 Average run time.

Figure 5 shows the partition number of the three different methods, and Figure 6 shows the average run time of the three methods. It is clear that the proposed method has the smallest partition number and least computational cost, followed by the distance partition method, and the K-means++ method has the biggest partition number and highest computational cost. The reason is that the clutter measurements are removed from the measurements by the density analysis technique, and the neighbor propagation technique is introduced to initially partition the measurements. However, for the K-means++ method, the way to set the $\mathrm{k}$ value is a problem, so the target number traversal technique is used. In this simulation, we also set $K \in\left(K_{L}, K_{U}\right)$. Notice that the partition number sharply decreases at time $50 \mathrm{~s}$; the reason is that the targets make a cross with each other, and thus the measurements mix and cannot be separated.

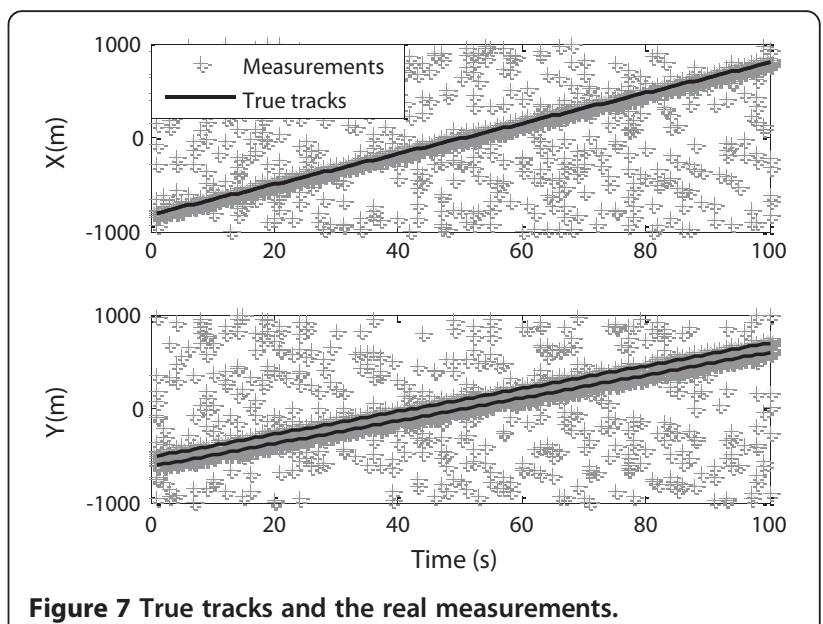




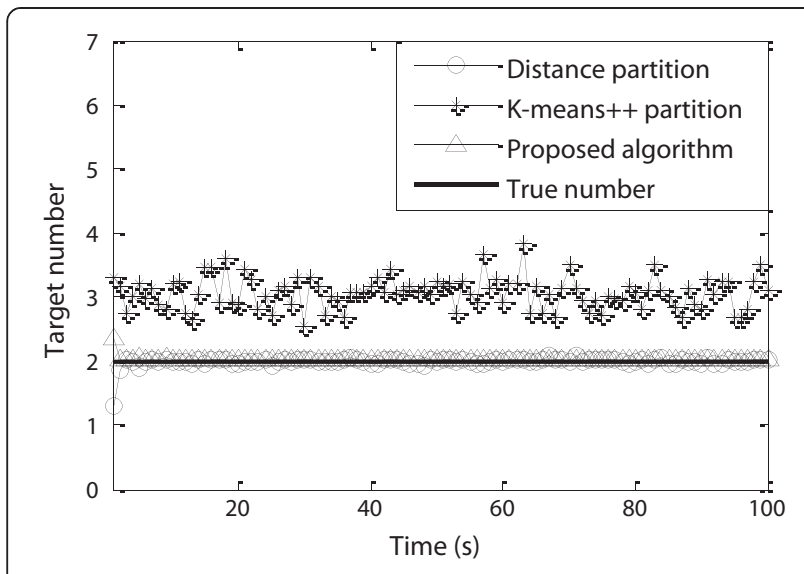

Figure 8 Target number estimates.

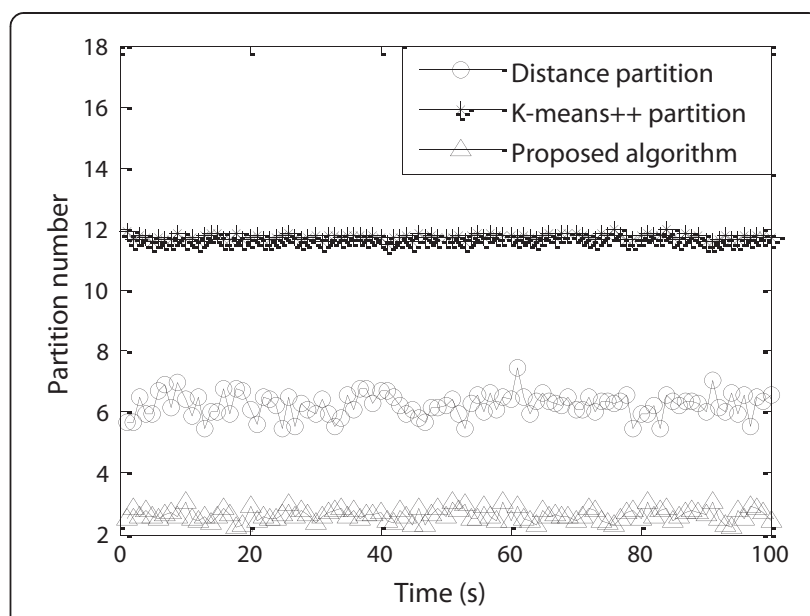

Figure 10 Partition number estimates.

partition and obviously better than that of the $\mathrm{K}$ means++ method.

\section{Conclusions}

For the METT in clutter, this paper introduces the spectral clustering technique to partition measurements and track the multiple extended target states using the ET-GM-PHD filter. First, we analyze the measurement density and remove the clutter from the measurements. Then, the spectral clustering method based on the neighbor propagation technique replaces the distance partition in the ET-GM-PHD algorithm. The simulation results show that the proposed algorithm has a better performance than the conventional methods, especially that it decreases the computational cost. Thus, the proposed algorithm has a good prospect in the engineering practice.

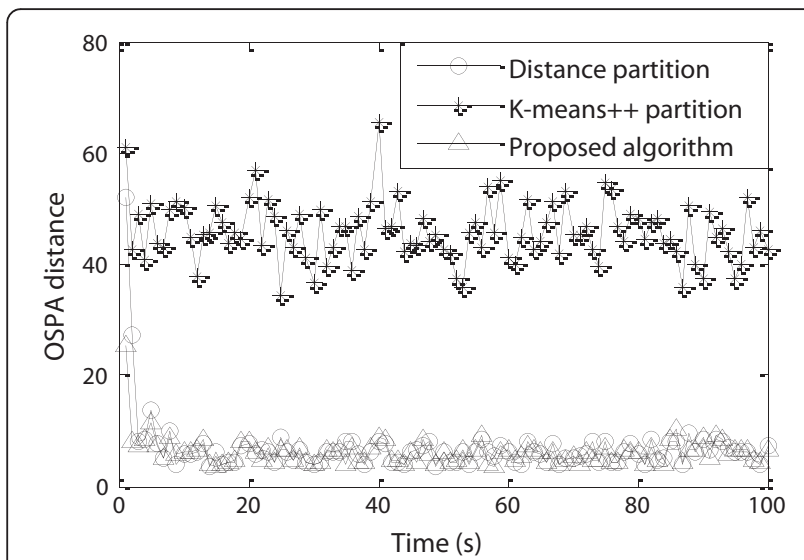

Figure 9 OSPA distance statistic.

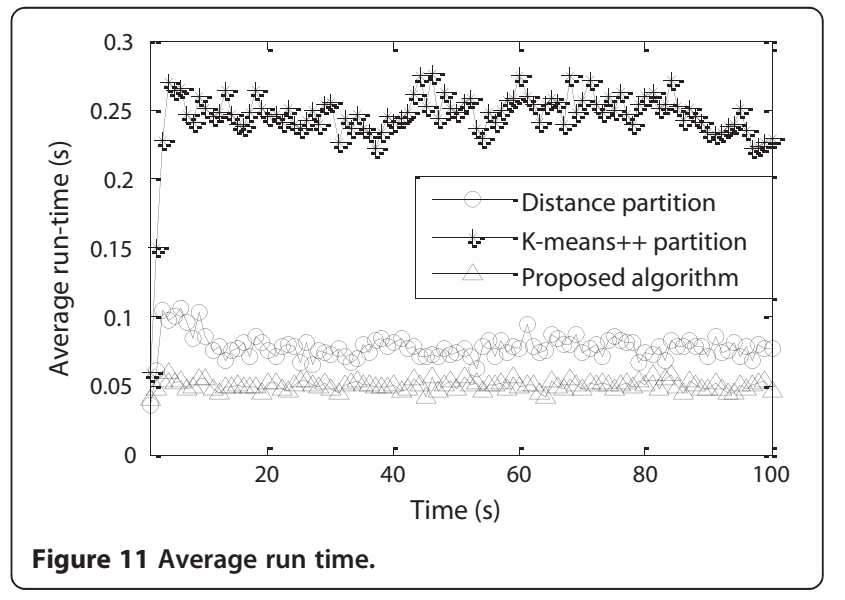




\section{Competing interests}

The authors declare that they have no competing interests.

\section{Acknowledgements}

This paper is supported by the National Natural Science Foundation of China (No. 61305017, 61304264) and the Natural Science Foundation of Jiangsu Province (No. BK20130154).

Received: 5 February 2014 Accepted: 14 July 2014

Published: 25 July 2014

\section{References}

1. T Fortmann, Y Bar-Shalom, M Scheffe, Sonar tracking of multiple targets using joint probabilistic data association. IEEE J. Ocean. Eng. 8(3), 173-183 (1983)

2. C Kreucher, K Kastella, AO Hero, Multitarget tracking using the joint multitarget probability density. IEEE Trans. Aero. Electron. Syst. 41(4), 1396-1414 (2005)

3. MR Morelande, CM Kreucher, K Kastella, A Bayesian approach to multiple target detection and tracking. IEEE Trans. Signal Process. 55(5), 1589-1604 (2007)

4. K Gilholm, S Godsill, S Maskell, D Salmond, Poisson models for extended target and group tracking, in Proceedings of Signal and Data Processing of Small Targets, California, USA, 2005

5. K Gilholm, D Salmond, Spatial distribution model for tracking extended objects. IEE Proceedings Radar, Sonar and Navigation 152(5), 364-371 (2005)

6. M Baum, UD Hanebeck, Random hypersurface models for extended object tracking, in Proceedings of the IEEE International Symposium on Signal Processing and Information Technology (Ajman, United Arab Emirates, 2009), pp. 178-183

7. M Baum, UD Hanebeck, Shape tracking of extended objects and group targets with star-convex RHMs, in Proceedings of the 14th International Conference on Information Fusion (IEEE, Chicago Illinois, USA, 2011), pp. 1-8

8. R Mahler, PHD filters for nonstandard targets, I: extended targets, in Proceedings of the 12th International Conference on Information Fusion (IEEE, Seattle, USA, 2009), pp. 915-921

9. K Granström, C Lundquist, U Orguner, A Gaussian mixture PHD filter for extended target tracking, in Proceedings of the 13th International Conference on Information Fusion (IEEE, Edinburgh, U.K., 2010), pp. 1-8

10. K Granström, C Lundquist, O Orguner, Extended target tracking using a Gaussian-mixture PHD filter. IEEE Trans. Aero. Electron. Syst. 48(4), 3268-3286 (2012)

11. U Orguner, C Lundquist, $K$ Granström, Extended target tracking with a cardinalized probability hypothesis density filter, in Proceedings of the 14th International Conference on Information Fusion (IEEE, Chicago, USA, 2011), pp. 1-8

12. K Granström, U Orguner, A PHD filter for tracking multiple extended targets using random matrices. IEEE Trans. Signal. Process. 60(11), 5657-5671 (2012)

13. C Lundquist, K Granström, U Orguner, An extended target CPHD filter and a gamma Gaussian inverse Wishart implementation. IEEE Journal of Selected Topics in Signal Processing 7(3), 472-483 (2013)

14. A Swain, D Clark, The PHD filter for extended target tracking with estimable extent shape parameters of varying size, in Proceedings of the 15th International Conference on Information Fusion (IEEE, Singapore, 2012), pp. 1111-1118

15. JW Koch, Bayesian approach to extended object and cluster tracking using random matrices. IEEE Trans. Aero. Electron. Syst. 44(3), 1042-1059 (2008)

16. JW Koch, M Feldmann, Cluster tracking under kinematical constraints using random matrices. Robot. Auton. Syst. 57(3), 296-309 (2009)

17. M Feldmann, D Fränken, JW Koch, Tracking of extended objects and group targets using random matrices. IEEE Trans. Signal Process. 59(4), 1409-1420 (2011)

18. R Mahler, Multi-target Bayes filtering via first-order multi-target moments. IEEE Trans. Aero. Electron. Syst. 39(4), 1152-1178 (2003)

19. BN Vo, WK Ma, The Gaussian mixture probability hypothesis density filter IEEE Trans. Signal Process. 54(11), 4091-4104 (2006)

20. P Braca, S Marano, V Matta, P Willett, Multitarget-multisensor ML and PHD: some asymptotics, in Proceedings of the 15th International Conference on Information Fusion (IEEE, Singapore, 2012), pp. 2347-2353

21. J Yang, $\mathrm{H}$ Ge, Adaptive probability hypothesis density filter based on variational Bayesian approximation for multi-target tracking. IET Radar, Sonar \& Navigation 7(9), 959-967 (2013)
22. P Braca, S Marano, V Matta, P Willett, Asymptotic efficiency of the PHD in multitarget/multisensor estimation. IEEE Journal of Selected Topics in Signal Processing 7(3), 553-564 (2013)

23. Zl Botev, JF Grotowski, DP Kroese, Kernel density estimation via diffusion. Ann. Stat. 38(5), 2916-2957 (2010)

24. D Comaniciu, P Meer, Mean shift: a robust approach toward feature space analysis. IEEE Trans. Pattern. Anal. Mach. Intell. 24(5), 603-619 (2002)

25. X Li, L Guo, Constructing affinity matrix in spectral clustering based on neighbor propagation. Neurocomputing 97(15), 125-130 (2012)

26. J Cao, P Chen, Q Dai, WK Ling, Local information-based fast approximate spectral clustering. Pattern Recogn. Lett. 38, 63-69 (2014)

27. D Schuhmacher, BT Vo, BN Vo, A consistent metric for performance evaluation of multi-object filters. IEEE Trans. Signal Process. 56(8), 3447-3457 (2008)

doi:10.1186/1687-6180-2014-117

Cite this article as: Yang et al:: Multiple extended target tracking algorithm based on GM-PHD filter and spectral clustering. EURASIP Journal on Advances in Signal Processing 2014 2014:117.

\section{Submit your manuscript to a SpringerOpen ${ }^{\circ}$ journal and benefit from:}

- Convenient online submission

- Rigorous peer review

- Immediate publication on acceptance

- Open access: articles freely available online

- High visibility within the field

- Retaining the copyright to your article

Submit your next manuscript at $>$ springeropen.com 